\title{
Anomalous length dependence of conductance of aromatic nanoribbons with amine anchoring groups
}

\author{
Ante Bilić* \\ CSIRO Mathematics, Informatics and Statistics, Private Bag 33, Clayton South 3169 VIC, Australia \\ Stefano Sanvito \\ School of Physics and CRANN, Trinity College, Dublin 2, Ireland \\ (Received 24 July 2012; published 6 September 2012)
}

\begin{abstract}
Two sets of aromatic nanoribbons, based around a common hexagonal scaffolding, with single and dual terminal amine groups have been considered as potential molecular wires in a junction formed by gold leads. Charge transport through the two-terminal device has been modeled using density functional theory (with and without self-interaction correction) and the nonequilibrium Green's function method. The effects of wire length, multiple terminal contacts, and pathways across the junction have been investigated. For nanoribbons with the oligopyrene motif and conventional single amine terminal groups, an increase in the wire length causes an exponential drop in the conductance. In contrast, for the nanoribbons with the oligoperylene motif and dual amine anchoring groups the predicted conductance rises with the wire length over the whole range of investigated lengths. Only when the effects of self-interaction correction are taken into account, the conductance of the oligoperylene ribbons exhibits saturation for longer members of the series. The oligoperylene nanoribbons, with dual amine groups at both terminals, show the potential to fully harness the highly conjugated system of $\pi$ molecular orbitals across the junction.
\end{abstract}

DOI: 10.1103/PhysRevB.86.125409 PACS number(s): 85.65.+h, 71.15.Mb, 72.80.Vp, 72.80.Le

\section{INTRODUCTION}

Recently, in an effort to identify a class of wires with charge transport properties suitable for use in molecular circuits, the conductance of polycyclic aromatic hydrocarbon (PAH) nanoribbons with pyrene and perylene motif has been investigated. ${ }^{1}$ The findings of a consistently high conductance, nearly independent of the anchoring structure, gold lead crystalline orientation, wire length, and bias voltage, makes the oligoperylene nanoribbons almost ideal wires for molecular circuitry. The thiol terminal groups, commonly utilized for the attachment of organic molecules to gold surfaces, provide a very strong bond with the leads. The nature of the S-Au bond is rather nonselective ${ }^{2}$ with regards to the binding site, which results in a large experimental uncertainty as to the atomic geometry of the interface. As a consequence, measured conductances typically exhibit an order of magnitude variation between experiments. ${ }^{3}$ The predicted conductances can exhibit even larger variations with the change in contact atomic geometry. ${ }^{4}$ In recent years, Venkataraman et al. proposed amine anchoring groups as an alternative to thiols. ${ }^{5}$ Junctions formed with amine-terminated molecules display well defined, reproducible conductance histograms, with a narrow spread. ${ }^{5}$ The success brought up by the amine-gold links is a consequence of the highly selective, yet flexible, bonding nature. The amine group is found to form bonds with undercoordinated gold atoms, such as isolated adatoms. ${ }^{5,6}$ This finding has motivated the studies of the effects of molecular length, ${ }^{5,6}$ conformation, and chemical substituents ${ }^{9}$ on the transport.

Here we report computational results on the conductance through two sets of aromatic nanoribbons, oligopyrenes, and oligoperylenes, as a function of the wire length and voltage bias. The two series share the same scaffolding of alternating double and single carbon rings along the transport axis and symmetry. The common monohydrogenated armchair edges provide the necessary chemical stability. ${ }^{10}$ The pyrene and perylene molecules, connected to the two-terminal device by the amine head groups, are shown in Fig. 1. While oligopyrenes terminate with single phenyl rings, oligoperylenes are terminated with double rings and, hence, dual amine head groups are introduced as the linkers at both ends of these wires.

\section{METHODS}

The computational approach closely takes after that previously utilized in the study of transport through the aromatic nanoribbons with thiol anchoring groups. ${ }^{1} \mathrm{~A} \mathrm{~N}$ pseudopotential of Troullier and Martins type, ${ }^{11}$ with a partial core correction, has been generated using the parameters available from URL http://www.eminerals.org/siesta. Geometry optimizations were performed using density functional theory (DFT) as implemented in the SIESTA program. ${ }^{12}$ Atomic geometries of all the junctions were fully relaxed via conjugate gradient optimization until the forces were smaller than $10 \mathrm{meV}^{-1}$. The LDA functional ${ }^{13}$ was used in both DFT and transport calculations. The range of the atomic orbitals was evaluated based on an energy increase of $1 \mathrm{mRy}$ arising due to the spherical confinement.

The optimized geometries obtained from the SIESTA computations, as well as all the relevant computational parameters, were subsequently employed in electron transport calculations. The latter were conducted using the nonequilibrium Green's function (NEGF) Landauer approach ${ }^{14}$ as implemented in the SMEAGOL package, ${ }^{15-17}$ which is interfaced to SIESTA. Selfinteraction correction (SIC) can be applied in combination with the LDA to the scattering region of the junction to partly rectify the artifacts arising from the band misalignment between the wire and terminals. ${ }^{18-20}$ Neither the local LDA 

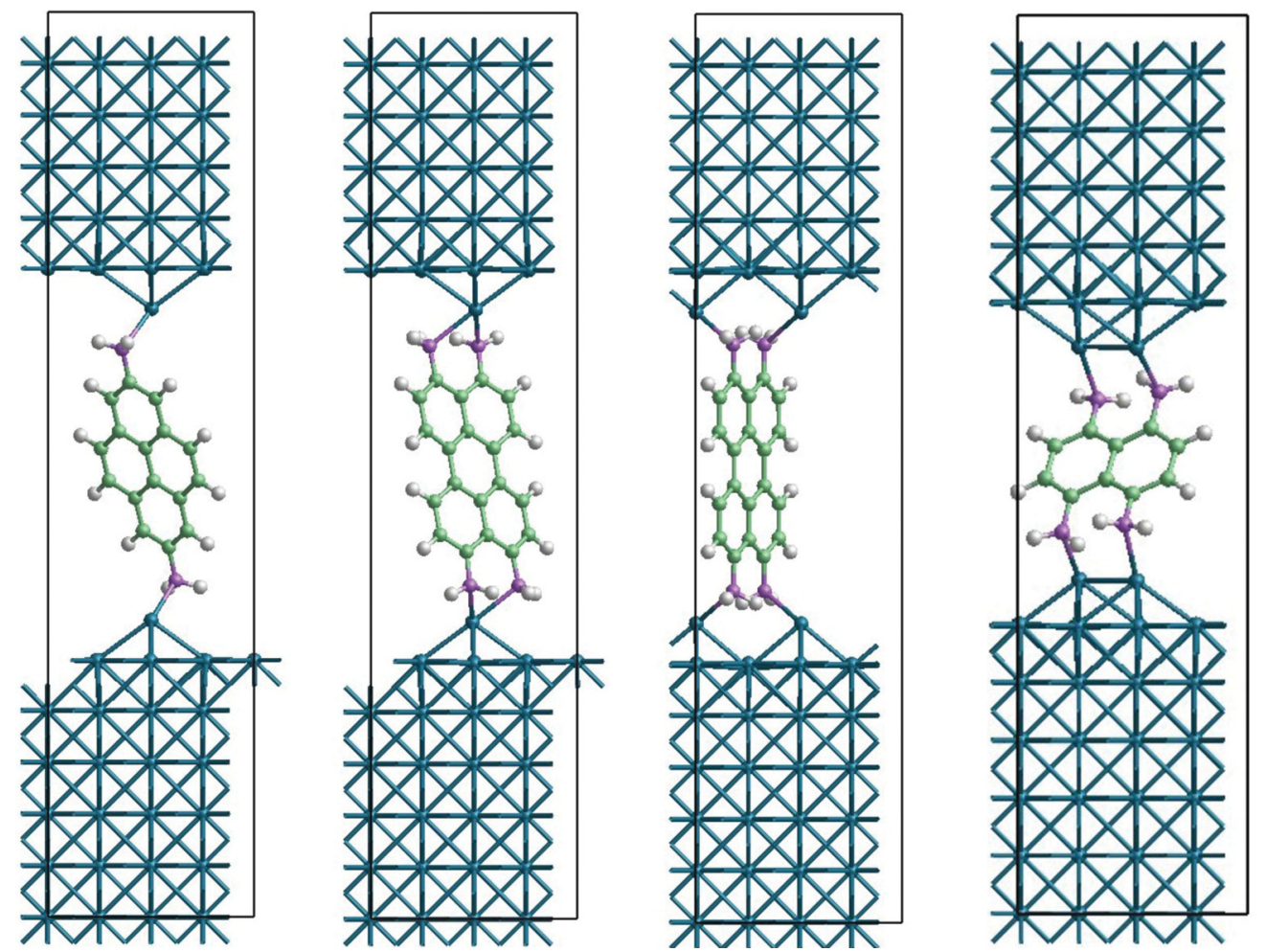

FIG. 1. (Color online) The 4R (pyrene diamine), 5R (perylene tetraamine), coordinated by single and dual Au adatoms, respectively, and $2 \mathrm{R}$ (napthalene tetraamine), coordinated by dual adjacent Au adatoms, in the two-terminal device.

or semilocal exchange-correlation functionals can predict $a$ priori the band alignment between the leads and wire with the necessary accuracy. Hence, there would be no reason to prefer either class of functional in this regard. However, the important difference is that self-interaction correction can be applied in combination with the LDA to the scattering region of the junction to partly rectify the artifacts arising from this misalignment. In the present work this has been done by utilizing the atomic self-interaction correction (ASIC). ${ }^{21,22} \mathrm{~A}$ value of 1.0 was used for the scaling parameter $\alpha$, amounting to the full ASIC. This approach gives an additional support to the present findings on transport by the investigated nanoribbons.

A junction of $\mathrm{Au}(001)$ terminated leads has been utilized to model the two-terminal device. The outer one or two layers of both leads were taken as parts of the extended molecule, with which they form the scattering region (this is the part treated at the NEGF self-consistent level). Even though the ammonia molecule can adsorb onto the flat low index gold surface atop an Au atom, ${ }^{23,24}$ owing to the highly reproducible results of the charge conductance through amine terminated wires, it has been postulated that the amine anchoring groups preferably make bonds with gold via isolated gold adatoms. ${ }^{25-27}$ This binding site selectivity reduces the relevance of the choice of the underlying crystalline orientation of the terminals. Hence, unlike in the case of thiolate terminated nanoribbons, ${ }^{1}$ it can be assumed that the present results equally apply to gold (111) terminals. The oligopyrene wires have been connected to the terminals by attaching the amine groups to the single adatoms on the opposite ends of the junction. In order to model the attachment of oligoperylenes, with the dual head groups, both the adsorption to the single and two gold adatoms on each side of the junction have been investigated. Figure 1 illustrates the optimized junction geometries comprising the four carbon ring (4R) pyrene and five ring (5R) perylene wires, the latter shown in the two considered conformations. The attachment via the single adatoms necessarily results in tilted conformations for both sets of the wires, owing to the approximately tetrahedral angles between the bonds coordinating the $\mathrm{N}$ atoms. In contrast, the attachment of the oligoperylenes via the dual gold adatoms, with each adatom being on the opposite side of the molecular plane as its neighbor, is particularly attractive because the molecular plane remains nearly perpendicular to the terminal surfaces. In this conformation the adatoms are separated by the second nearest neighbor distance. An alternative, in which the two adatoms are located on the nearest neighbor sites (akin to contact motif II in Ref. 25), has also been considered, shown in Fig. 1 for naphthalene in the junction. However, it has been found to be energetically less favorable (in the case of naphthalene by $0.637 \mathrm{eV}$ ) than the attachment via the second nearest neighbors, with transport results showing little difference between the two configurations.

\section{RESULTS AND DISCUSSION}

The $I-V$ characteristics and zero-bias transmission coefficients for pyrene (4R) and its seven (7R) and ten (10R) ring oligomers are shown in Fig. 2. The results for the transport through the single 1,4-benzenediamine ring (labeled $1 \mathrm{R})$ are also shown therein. It has been included in the oligopyrene set because it shares the single amine terminal groups with them and it can be considered to be the base 

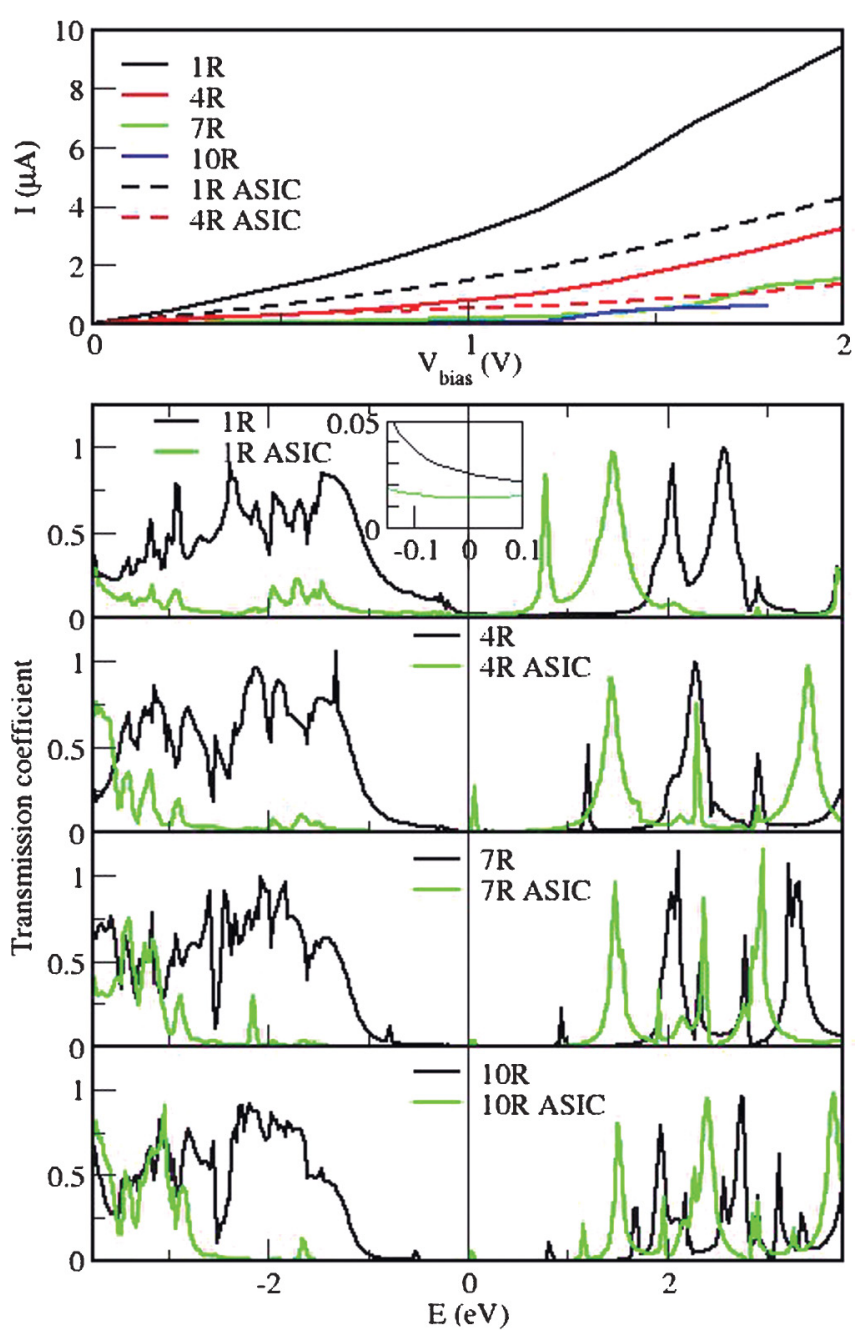

FIG. 2. (Color online) Upper panel: Current vs bias voltage for the 1-4-7-10R series of wires between the $\mathrm{Au}(001)$ terminals. Lower panels, from top to bottom: The zero-voltage transmission probability for the $1 \mathrm{R}, 4 \mathrm{R}, 7 \mathrm{R}$, and $10 \mathrm{R}$ nanoribbons, respectively. The energy is given relative to the Fermi level. Details of the transmission probability around the Fermi level for $1 \mathrm{R}$ wire are shown in the inset.

member of the 1-4-7-10R series. The transmission spectrum for $1 \mathrm{R}$ is in very good agreement with that of Quek et al., shown in Fig. 3 of Ref. 25, despite the differences in the surface indices, number and arrangement of Au adatoms, and computational methodologies. A closely resembling spectrum has also been evaluated in Ref. 26. The peak below the Fermi level and two peaks above it correspond to frontier molecular orbitals (MOs) of the 1,4-benzenediamine, highest occupied (HOMO), lowest unoccupied (LUMO), and second lowest unoccupied $(\mathrm{LUMO}+1)$. Low bias conductance exhibits the familiar exponential decay with the length of wire. ${ }^{28}$ An attenuation factor $\beta$ of $0.34 \AA^{-1}$ is obtained for this series, in reasonably good agreement with $0.25^{1}$ and $0.38^{28}$ previously evaluated for their counterparts with thiol head groups anchored to the $\mathrm{Au}(111)$ surface. The exponential decay of the conductance with the wire length, commonly observed for amine terminated molecules, ${ }^{5-7}$ is consistent with the nonresonant tunneling mechanism or superexchange. For $1 \mathrm{R}$ a zero-bias conductance of $0.026 G_{0}\left(e^{2} / h\right)$ is obtained,

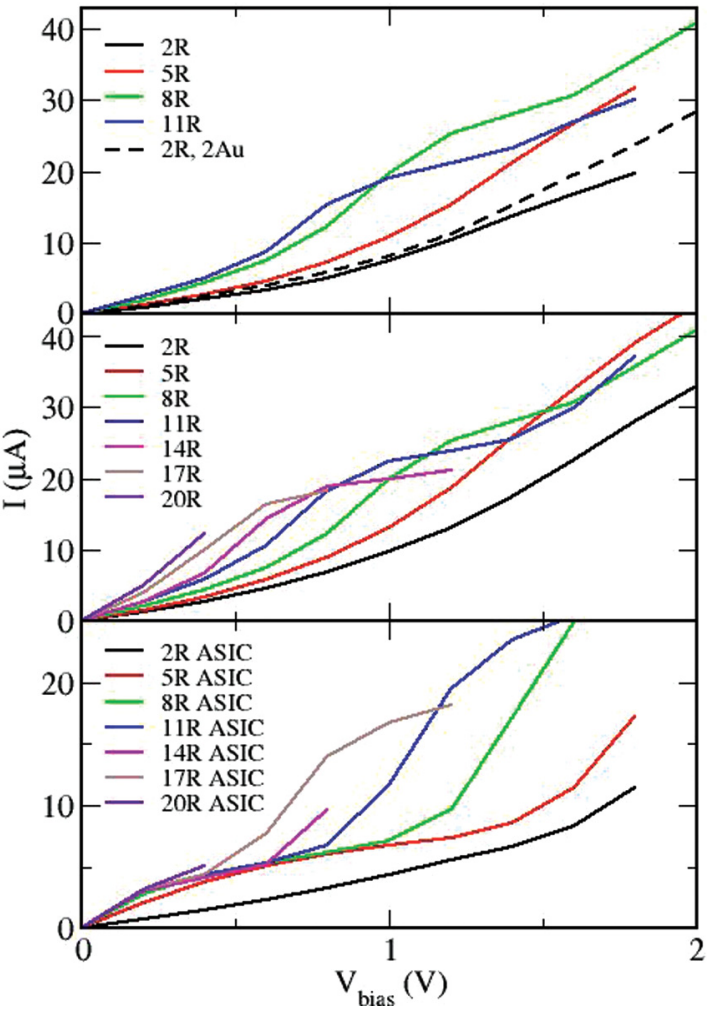

FIG. 3. (Color online) Upper panel: Current vs bias voltage for the 2-5-8-11R series of wires, coordinated via single Au adatoms by the terminals. Middle panel: Current vs bias voltage for the 2-5-811-14-17-20R series of wires, coordinated via dual Au adatoms by the terminals. Lower panel: The same as in the middle panel, after application of the ASIC.

four times larger than the measured value of $0.0064 G_{0} .{ }^{5}$ However, after the LDA electronic structure is corrected with the ASIC method, the predicted value is $0.014 G_{0}$, a factor of 2 above the experimental counterpart. This represents a rather impressive agreement between theory and measurements. In general, the findings for the amine terminated oligopyrene series are analogous to those for their thiolated counterparts, shown in Fig. 2 of Ref. 1, although with much smaller current magnitudes, owing to the much weaker coupling of amines to the contacts. In both cases the transmission spectra exhibit a widening gap between occupied and unoccupied transport channels as the length of the wire increases. The inclusion of ASIC causes a significant downshift of the energy levels of the frontier occupied states away from the Fermi level. This, in effect, dramatically reduces their contribution to the transmission. Consequently, the corrected gap is much wider.

The $I-V$ characteristics for perylene (5R) and its eight (8R) and 11 ring $(11 \mathrm{R})$ oligomers, coordinated to the contacts via single $\mathrm{Au}$ adatoms are shown in the upper panel of Fig. 3, together with the results for naphthalene (2R). Similar to $1 \mathrm{R}$ and oligopyrenes, $2 \mathrm{R}$ has been included in the oligoperylene set because of the common dual head groups and is considered to be the base member of the 2-5-8-11R series. Contrary to one's intuition, here the low bias current rises with the increasing wire length. This is also in contrast with the results for the same, but thiol terminated nanoribbons, which demonstrate nearly length-independent conductance. ${ }^{1}$ 
The same plot also shows the effect of coordination by dual adjacent $\mathrm{Au}$ adatoms on the conductance of $2 \mathrm{R}$. The corresponding current is only about $10 \%-15 \%$ higher than that when single adatoms are employed. Even though the adatoms can be considered as a part of the extended wire, single adatoms, unlike the single head groups, do not appear to pose a sizable bottleneck to the charge flow. The middle panel of Fig. 3 shows the $I-V$ characteristics of the perylene series coordinated via the dual, separated Au adatoms. The current exhibits a further increase, about $25 \%-35 \%$ over that of the same wire in the single adatom coordination by the contacts. Clearly the separated dual adatoms provide not only an energetically more favorable attachment to the contacts than the adjacent adatoms, but also an enhanced conductance. The unusual increase of the low bias current with length is clearly manifested also in this conformation. To further explore the anomalous and seemingly unphysical trend, the nanoribbon length has been extended to include the 14 (14R), 17 (17R), and 20 (20R) ring oligopreylenes, pushing the computational effort to the limits of feasibility. However, the calculated low bias current still exhibits a uniform rise across the entire 2-20R oligoperylene set. Eventually, as the lower panel of Fig. 3 shows, upon the inclusion of the ASIC the current still rises across the $2 \mathrm{R}-11 \mathrm{R}$ subset, where it reaches saturation at about $3 \mu \mathrm{A}$ for a bias of $0.2 \mathrm{~V}$. The effect of the ASIC is also manifested in the largely reduced current magnitudes, unlike in the case of thiol terminated nanoribbons (shown in Fig. 3 of Ref. 1). Note that, because the self-consistent convergence becomes increasingly more difficult to achieve for longer wires and in particular with the higher voltage bias, the current for the 14-20R wires has been calculated only in a low bias range.

The zero-bias transmission coefficients for the perylene series are shown in Fig. 4. In contrast to those of the pyrene series, here the gap between the occupied and unoccupied channels closes with the increasing wire length and conjugation. A similar observation has been made in the case of thiol terminated oligoperylenes. ${ }^{1}$ The weaker nature of the amine-gold bond, however, results in certain differences, which are manifested through more discrete transmission spectra with narrow resonances, their energy alignment with respect to the Fermi level and a larger impact of the ASIC than in the case of thiol head groups.

In order to fully understand the anomalous conductance of the perylene nanoribbons it is useful to consider the infinite polymer with the same hexagonal scaffolding, whose unit cell is shown in the inset of Fig. 5, which also shows the frontier electronic states: second HOMO (HOMO-1), HOMO, LUMO, and second LUMO (LUMO + 1). The plot of energy bands indicates that the wire is almost metallic, with a band gap at the $\Gamma$ point close to zero. The "conical" (or, rather, linear) dispersion away from the Dirac point is an indication of the graphenelike, although one dimensional, nature of the wire. ${ }^{29}$ The HOMO and LUMO at the zone center are near degenerate and, in addition, they are both antisymmetric with respect to the mirror plane $\sigma_{\mathrm{v}}$ perpendicular to the wire. Because of this symmetry their orbital weights on the central atoms are zero. If the wire is terminated with single rings, as in the case of oligopyrenes, the single head groups, also situated on the axis, can at best provide a weak, through space, coupling of these MOs to the contacts. Hence, despite the

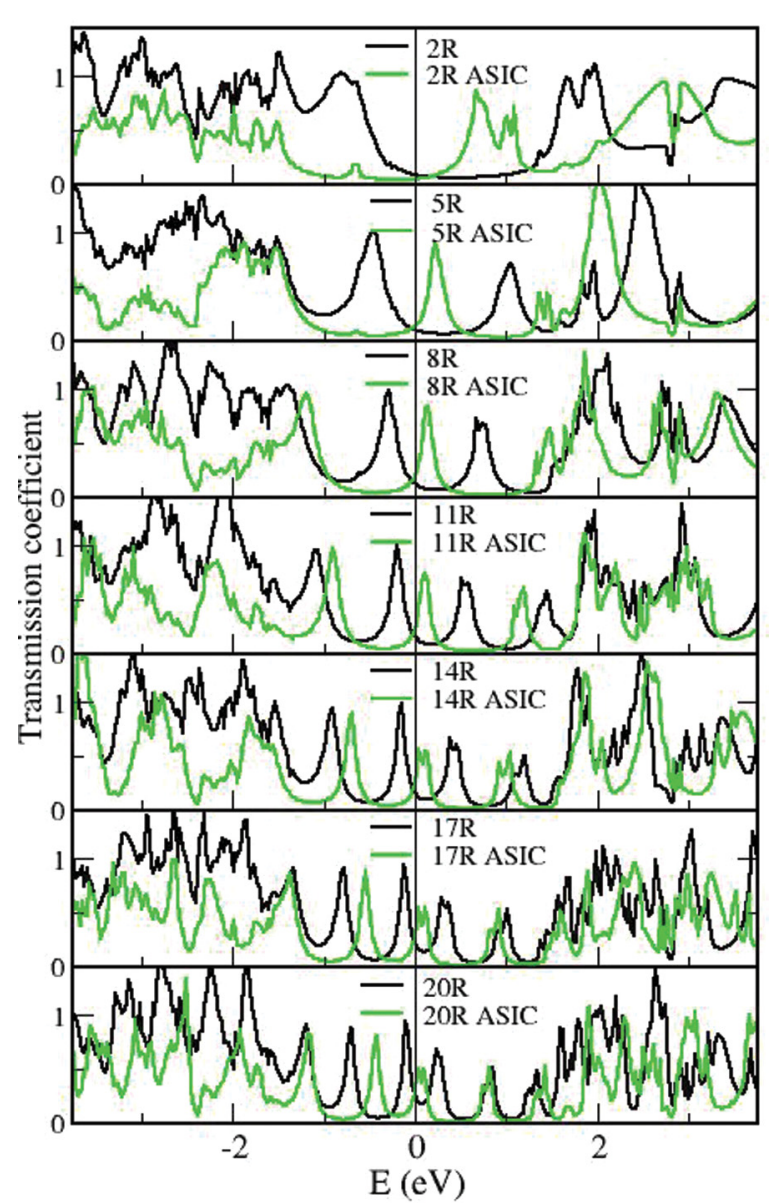

FIG. 4. (Color online) Panels show, from top to bottom, the zerovoltage transmission probability for the $2 \mathrm{R}, 5 \mathrm{R}, 8 \mathrm{R}, 11 \mathrm{R}, 14 \mathrm{R}, 17 \mathrm{R}$, and $20 \mathrm{R}$ nanoribbons, respectively. The energy is given relative to the Fermi level.

reduced HOMO-LUMO gap with the increasing wire length and conjugation, these two most important MOs do not make a substantial contribution to the transmission, which shows an increasingly large gap. In contrast, oligoperylenes are more electron rich than the pyrenes of the same length, they have an additional ring and exhibit a smaller HOMO-LUMO gap, and can effectively couple these antisymmetric orbitals to the contacts via the dual head groups which are positioned away from the symmetry axis. This feature gives rise to the increasing number of conducting channels around the Fermi level in the transmission spectrum as the wire length and conjugation are extended. In case of the thiol head groups, the benefits of the enhanced density of states in the Fermi level proximity appear to merely cancel out the detrimental effects of the increased tunneling length. For the amine head groups, the present findings suggest that benefits outweigh the penalty.

In order to gain a further insight into the nature of the amine-gold bond the transmission probability for the $1 \mathrm{R}$ wire has been replotted in Fig. 6, together with the projected density of states (PDOS) of an edge $\mathrm{C}$ atom, a $\mathrm{C}$ atom on the molecular axis, and a $\mathrm{N}$ atom. Only the components of the PDOS originating from the $p$-type atomic orbitals which contribute to the out-of-plane $\pi$-type MOs are shown. These make the largest contribution to the transport channels for the 


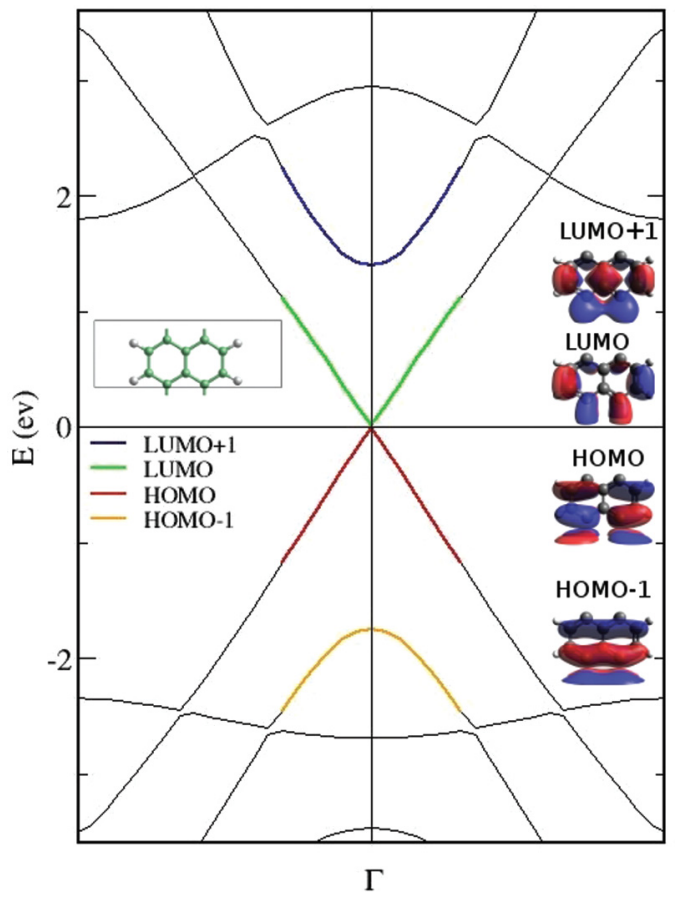

FIG. 5. (Color online) Energy bands of the electronic states of the wire whose unit cell is shown in the inset. Only the states in the vicinity of the Fermi level are shown. The states corresponding to the frontier MOs are highlighted around the zone center. The orbital shapes of these states at the zone center are also shown.

planar, highly conjugated molecular wires. ${ }^{30}$ The first peak in the transmission spectrum below the Fermi level is a product of the 1R HOMO, which is symmetric, with all $\mathrm{C}$ and $\mathrm{N}$ atoms contributing to its $\pi$ system, as also demonstrated in Fig. 4 of Ref. 26. The LUMO is an antisymmetric $\pi$ system, to which only the edge $\mathrm{C}$ atoms of $1 \mathrm{R}$ contribute. This is the reason behind the very narrow resonance in the spectrum, indicating a weak through-space coupling to gold. A similar peak is seen at $-3 \mathrm{eV}$ below the Fermi level (labeled HOMO-4 in Ref. 26). Evidently the two peaks at $\pm 2.5 \mathrm{eV}$ from the Fermi level are again the products of symmetric MOs, with orbital weights on all $\mathrm{C}$ and $\mathrm{N}$ atoms. Once the ASIC is applied, however, the energies of the occupied frontier MOs are shifted by about $-2.5 \mathrm{eV}$, leaving a large gap in the transmission. This is partly offset by a smaller shift of about $-1 \mathrm{eV}$ of the LUMO and LUMO + 1 , whose tails now make the key contributions to the spectrum at the Fermi level. Note that the downshift of the unoccupied energy levels is partly an artifact of the ASIC since one does not expect a drift of empty states when the self-interaction is removed from the filled electronic states. Therefore, it appears that the conductance through the $1 \mathrm{R}$ junction takes place primarily via electrons, rather than holes. This finding is contrary to the previous interpretations found in the literature ${ }^{25-27}$ and it further stresses the importance of the accurate SIC for DFT-based charge transport investigations on nanostructures. While Quek et al. also considered the effects of the SIC, albeit in a heuristic manner, and downshifted the HOMO energy by a comparable amount of $-2 \mathrm{eV}$, in their work an upward energy shift by $+2 \mathrm{eV}$ was applied to the LUMO. The resulting HOMO-LUMO gap of $4.8 \mathrm{eV}$ is a

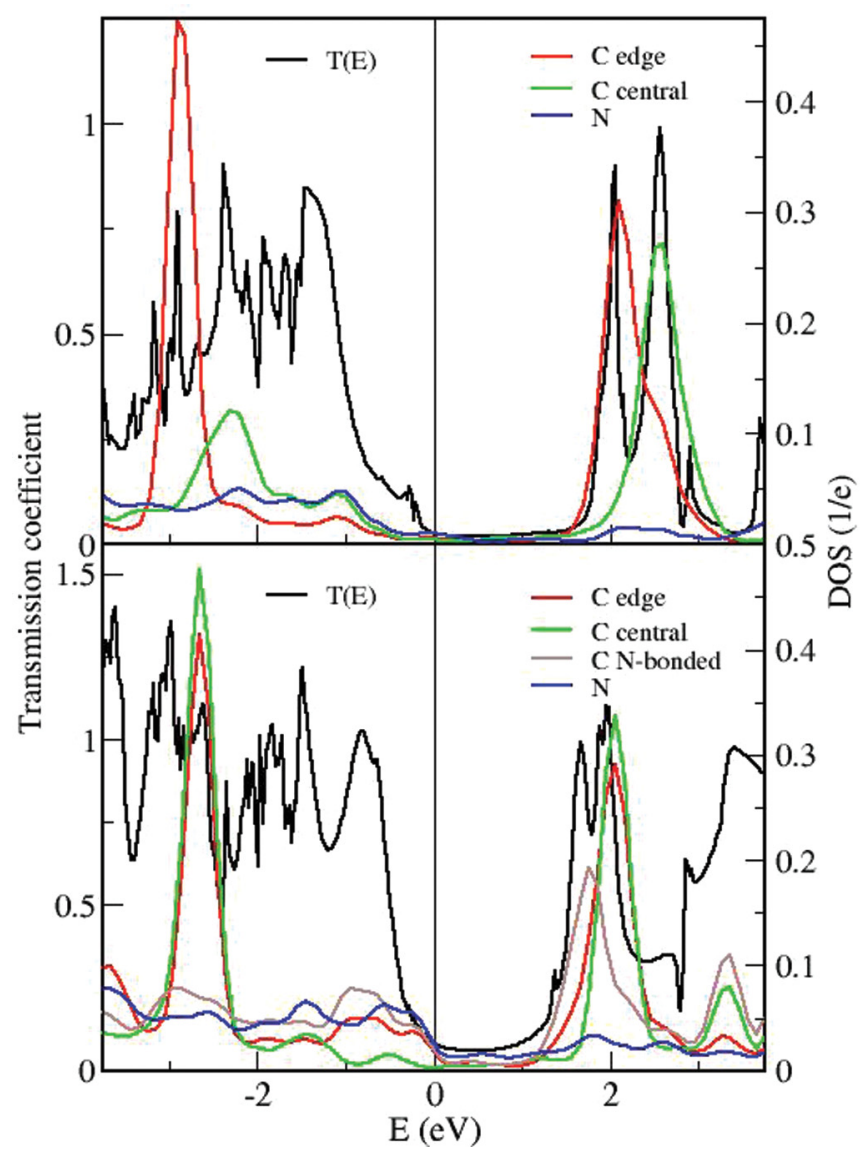

FIG. 6. (Color online) Upper panel: The zero-voltage transmission probability $T(E)$ for the $1 \mathrm{R}$ junction. The out-of-plane $p$ orbital contribution to the partial density of states (PDOS) from an edge $\mathrm{C}$ atom, a central $\mathrm{C}$ atom, and $\mathrm{N}$ atom are also shown. Lower panel: Similar as in the upper panel for the $2 \mathrm{R}$ junction. Only the ASIC-uncorrected results are shown for both $1 \mathrm{R}$ and $2 \mathrm{R}$.

very reasonable estimate for a gas phase 1,4-benzenediamine, but it is far from certain that the same gap can be expected when it is bonded to the contacts. ${ }^{31}$ Based on the present ASIC calculations, however, there is no evidence of an upward LUMO energy correction and, indeed, this is not expected at all from the SIC. In fact, as the transmission spectra in Fig. 2 show, the closer the energies of the unoccupied channels are to the Fermi before the application of ASIC, the more downshifted they are upon the correction. Analogous observations can be made for the oligoperylene spectra in Fig. 4.

The corresponding PDOS contribution to the $2 \mathrm{R}$ transmission spectrum is shown in the lower panel of Fig. 6. There are three kinds of $\mathrm{C}$ atoms to consider in this wire: the central, the edge, and the $\mathrm{N}$ coordinated. Unlike the case of $1 \mathrm{R}$, both the HOMO and LUMO, whose orbital densities closely match those of the infinite wire in Fig. 4, are antisymmetric. This is manifested by the lack of significant DOS on the central C atom, which contributes substantially only to the channels at 2 and $-2.7 \mathrm{eV}$. The dominant contribution to the HOMO and LUMO channels comes from the $\mathrm{N}$ and the $\mathrm{N}$-coordinated $\mathrm{C}$ atom, thus confirming the effective harnessing of the antisymmetric MOs by the dual head groups away from the molecular axis. The effect of the ASIC on the $2 \mathrm{R}$ transmission 
spectrum is very similar to that of the $1 \mathrm{R}$ junction, resulting in a somewhat smaller gap, but even more prominent transport via the unoccupied channels.

The symmetries of the frontier MOs of the longer counterparts in the two nanoribbon series are the same as those of $1 \mathrm{R}$ and $2 \mathrm{R}$. A PDOS analysis for the 4R contribution to the transmission (not shown), indicates that the gap between frontier MO energies is smaller, as expected, than in the case of $1 \mathrm{R}$, yet the gap in the transmission appears wider. A careful inspection of the trend in the spectra presented in Fig. 2 shows that the energies of the unoccupied channels are getting lower with the increased wire length. However, their widths and amplitudes drop rapidly as they approach the Fermi level. The magnitude of the HOMO peak channel also drops substantially across the series. Therefore, the widening transmission gap is simply a result of a relatively ineffective frontier MO conjugation across the junction, which, in turn, is mostly a consequence of the single terminal groups. This is the true reason behind the detrimental length effect on the pyrenes.

The PDOS analysis of the 5R junction (not shown) suggests a completely analogous interpretation of the transport contributions from the antisymmetric HOMO and LUMO as that given for the $2 \mathrm{R}$. These are found to give rise to the two prominent peaks around the Fermi level. The application of the ASIC results in the HOMO energy being downshifted away from the Fermi level to the edge of the broad continuum at about $-1.5 \mathrm{eV}$, where it does not feature as an isolated peak. In contrast, the corrected LUMO channel is situated even closer to the Fermi energy, giving rise to an even more electronic (as opposed to holelike without the ASIC) nature of conductance. It is very interesting to observe the trend in Fig. 4 with the extended length and conjugation. As the HOMO-LUMO gap closes for both corrected and uncorrected spectra, the latter consistently show the HOMO channel being closer to the Fermi level. An opposite effect is seen for the corrected spectra, with a dominant LUMO channel just above the Fermi level. Coincidentally, the HOMO peak of the uncorrected spectra and LUMO of their corrected counterparts intersect almost exactly at the Fermi level, resulting in an almost identical zero-bias conductance (given by the amplitude at this point), regardless of the application of the ASIC. This finding gives an additional weight to the present results. The important difference, however, is the electron carrier nature of transport implied by the ASIC application.

Similar to their thiolated counterparts, amine-terminated perylene nanoribbons exhibit very desirable transport proper- ties, with similarly consistent high currents, although much more sensitive to the bias voltage. Several thiol-terminated wires, such as perylenes and polyynes ${ }^{32}$ have been predicted to exhibit a length independent conductance. (In fact, for polyynes an increase in the current with the length has been reported, ${ }^{33}$ but the corrected band alignment eventually restored the trend of decreasing conductance vs length). The much weaker amine-gold bond, although very selective and flexible, is not particularly suitable for molecular circuitry, where stronger, more stable bonding is required. However, the present findings on the unusual length dependence of the nanoribbon conductance, provided in part by this weak bond, deserve a further interest and will, hopefully, stimulate an experimental effort to validate them. It is important to remember that the predicted anomalous conductance could be observed only in the very low temperature limit, without inelastic scattering and phase decoherence. At an elevated temperature phonon scattering would affect the conductance of longer nanoribbons more than that of the short ones and, effectively, lift the predicted anomaly.

\section{CONCLUSIONS}

In summary, we have investigated the conductance of two sets of aromatic nanoribbons, with the common honeycomb scaffolding. The effects of molecule length and multiple amine head groups on charge transport have been investigated. The increase in the wire length has been confirmed to cause an exponential reduction in the conductance of the oligopyrene class of nanoribbons, with single amine groups at both terminals. In contrast, for the oligoperylene nanoribbons an anomalous length dependence is observed with conductance uniformly rising with the wire length. The application of self-interaction correction eventually results in a saturating low bias conductance.

\section{ACKNOWLEDGMENTS}

This work was supported by the Flexible Electronics Theme of the CSIRO Future Manufacturing Flagship. A.B. thanks the CSIRO for support through the Julius Career Award. The use of the NCI National Facility supercomputers at the ANU is gratefully acknowledged. The SMEAGOL project is sponsored by Science Foundation of Ireland (Grant No. 07/IN/I945), by KAUST (FIC/2010/08) and by CRANN.

\footnotetext{
*Corresponding author: ante.bilic@csiro.au

${ }^{1}$ A. Bilić, J. D. Gale, and S. Sanvito, Phys. Rev. B 84, 205436 (2011); 86, 039905(E) (2012).

${ }^{2}$ A. Bilić, J. R. Reimers, and N. S. Hush, J. Chem. Phys. 122, 094708 (2005).

${ }^{3}$ S. M. Lindsay and M. A. Ratner, Adv. Mater. 19, 23 (2007).

${ }^{4}$ K.-H. Müller, Phys. Rev. B 73, 045403 (2006).

${ }^{5}$ L. Venkataraman, J. E. Klare, I. W. Tam, C. Nuckolls, M. S. Hybertsen, and M. L. Steigerwald, Nano Lett. 6, 458 (2006).
}

${ }^{6}$ S. Y. Quek, H. J. Choi, S. G. Louie, and J. B. Neaton, Nano Lett. 9 , 3949 (2009).

${ }^{7}$ L. Venkataraman, J. E. Klare, C. Nuckolls, M. S. Hybertsen, and M. L. Steigerwald, Nature (London) 442, 904 (2006).

${ }^{8}$ S. Y. Quek, M. Kamenetska, M. L. Steigerwald, H. J. Choi, M. S. Hybertsen, J. B. Neaton, and L. Venkataraman, Nat. Nanotechnol. 4, 230 (2009).

${ }^{9}$ L. Venkataraman, Y. S. Park, A. C. Whalley, C. Nuckolls, M. S. Hybertsen, and M. L. Steigerwald, Nano Lett. 7, 502 (2008). 
${ }^{10}$ T. Wassmann, A. P. Seitsonen, A. M. Saita, M. Lazezeri, and F. Mauri, Phys. Rev. Lett. 101, 096402 (2008).

${ }^{11}$ N. Troullier and J. L. Martins, Phys. Rev. B 43, 1993 (1991).

${ }^{12}$ J. M. Soler, E. Artacho, J. D. Gale, A. García, J. Junquera, P. Ordejón, and D. Sánchez-Portal, J. Phys.: Condens. Matter 14, 2745 (2002).

${ }^{13}$ D. M. Ceperley and B. J. Alder, Phys. Rev. Lett. 45, 566 (1980).

${ }^{14}$ P. S. Damle, A. W. Ghosh, and S. Datta, in Molecular Nanoelectronics; edited by M. A. Reed and T. Lee (American Scientific, Los Angeles, 2003).

${ }^{15}$ A. R. Rocha, V. M. Garcia-Suarez, S. W. Bailey, C. J. Lambert, J. Ferrer, and S. Sanvito, Nat. Mater. 4, 335 (2005).

${ }^{16}$ A. R. Rocha, V. M. Garcia-Suarez, S. W. Bailey, C. J. Lambert, J. Ferrer, and S. Sanvito, Phys. Rev. B 73, 085414 (2006).

${ }^{17}$ I. Rungger and S. Sanvito, Phys. Rev. B 78, 035407 (2008).

${ }^{18}$ C. Toher, A. Filippetti, S. Sanvito, and K. Burke, Phys. Rev. Lett. 95, 146402 (2005).

${ }^{19}$ C. Toher and S. Sanvito, Phys. Rev. Lett. 99, 056801 (2007).

${ }^{20}$ C. Toher and S. Sanvito, Phys. Rev. B 77, 155402 (2008).

${ }^{21}$ C. D. Pemmaraju, T. Archer, D. Sánchez-Portal, and S. Sanvito, Phys. Rev. B 75, 045101 (2007).
${ }^{22}$ A. Filippetti, C. D. Pemmaraju, S. Sanvito, P. Delugas, D. Puggioni, and V. Fiorentini, Phys. Rev. B 84, 195127 (2011).

${ }^{23}$ A. Bilić, J. R. Reimers, N. S. Hush, and J. Hafner, J. Chem. Phys. 116, 8981 (2002).

${ }^{24}$ S. Piana and A. Bilic, J. Phys. Chem. B 110, 23467 (2006).

${ }^{25}$ S. Y. Quek, L. Venkataraman, H. J. Choi, S. G. Louie, M. S. Hybertsen, and J. B. Neaton, Nano Lett. 7, 3477 (2007).

${ }^{26}$ J. Ning, R. Li, X. Shen, Z. Qian, S. Hou, A. R. Rocha, and S. Sanvito, Nanotechnology 18, 345203 (2007).

${ }^{27}$ M. S. Hybertsen, L. Venkataraman, J. E. Klare, A. C. Whalley, M. L. Steigerwald, and C. Nuckolls, J. Phys.: Condens. Matter 20, 374115 (2008).

${ }^{28}$ H. Liu, C. Yu, N. Gao, and J. Zhao, Chem. Phys. Chem. 11, 1895 (2010).

${ }^{29}$ A. H. Castro Neto, F. Guinea, N. M. R. Peres, K. S. Novoselov, and A. K. Geim, Rev. Mod. Phys. 81, 109 (2009).

${ }^{30}$ A. Bilić, Ž. Crljen, B. Gumhalter, J. D. Gale, I. Rungger, and S. Sanvito, Phys. Rev. B 81, 155101 (2010).

${ }^{31}$ J. B. Neaton, M. S. Hybertsen, and S. G. Louie, Phys. Rev. Lett. 97, 216405 (2006).

${ }^{32}$ Ž. Crljen and G. Baranović, Phys. Rev. Lett. 98, 116801 (2007).

${ }^{33}$ V. M. García-Suárez and C. J. Lambert, Nanotechnology 19, 455203 (2008). 